\title{
Schwinger Pair Production at Finite Temperature in Scalar QED
}

\author{
Sang Pyo Kin? \\ Department of Physics, Kunsan National University, Kunsan 573-701, Korea and \\ Asia Pacific Center for Theoretical Physics, Pohang 790-784, Korea \\ Hyun Kyu Leđ丹 \\ Department of Physics and BK21 Division of Advanced Research \\ and Education in Physics, Hanyang University, Seoul 133-792 and \\ Asia Pacific Center for Theoretical Physics, Pohang 790-784, Korea
}

(Dated:)

\begin{abstract}
In scalar QED we study the Schwinger pair production from an initial ensemble of charged bosons when an electric field is turned on for a finite period together with or without a constant magnetic field. The scalar QED Hamiltonian depends on time through the electric field, which causes the initial ensemble of bosons to evolve out of equilibrium. Using the Liouville-von Neumann method for the density operator and quantum states for each momentum mode, we calculate the Schwinger pair-production rate at finite temperature, which is the pair-production rate from the vacuum times a thermal factor of the Bose-Einstein distribution.
\end{abstract}

PACS numbers: 12.20.-m, 13.40.-f, 11.10.Wx

\section{INTRODUCTION}

Vacuum polarization and pair production has been known more than seven decades ago [1]. Schwinger used the proper-time method to find the exact one-loop effective action for a uniform electromagnetic field [2]. Since then vacuum polarization and Schwinger pair production has been investigated more or less from a theoretical view point (for a review on recent development of strong QED, see Ref. [3]). However, recently the study of strong QED is more physically motivated than ever since it will have many interesting physical applications in the near future. In the near future terrestrial experiments, the x-ray free electron lasers from Linac Coherent Light Source at SLAC and the TeV Energy Superconducting Linear Accelerator at DESY are expected to achieve electromagnetic fields near the QED regime [4, 5, 6]. In astrophysics, most neutron stars are expected to have magnetic fields comparable to the critical strength $B_{c}=m^{2} c^{2} / e \hbar\left(4.4 \times 10^{13} G\right.$ for electrons $)$ and furthermore some magnetars are known to have magnetic fields a few order above the critical strength. It is interesting that the ratio of the pair-production rate in the presence of parallel electric and magnetic fields to that in the pure electric field is $\mathcal{N}(E, B) / \mathcal{N}(E, 0)=(\pi q B / q E) \times \operatorname{coth}(\pi q B / q E)$. This implies that the pair-production rate is enhanced by the strong magnetic field, though the leading behavior is still determined by the electric field as $\mathcal{N}(E, 0)=(q E)^{5 / 2} e^{-\pi m^{2} / q E} /\left(4 \pi^{3} m\right)$. In these compact stars pairs would be produced at non-zero temperature.

Another interesting issue from the view point of strong QED and astrophysics is strange quark stars, hypothetical astrophysical objects. Witten proposed that strange quark matter might be a stable state of nuclear matter at ultrahigh density, which was numerically studied by Farhi and Jaffe [7]. Based on this hypothesis strange quark stars were proposed as an alternative to neutron stars [8]. The charge neutrality and chemical equilibrium of strange quark stars would require a net amount of electrons, which are bounded by the Coulomb attraction from the positive core and form an electrosphere on the surface. The Coulomb barrier from the electrosphere can generate an electric field as large as $5 \times 10^{17} \mathrm{~V} / \mathrm{cm}$, which is stronger than the critical strength $E_{c}=m^{2} c^{3} / \mathrm{e} \hbar\left(1.3 \times 10^{16} \mathrm{~V} / \mathrm{cm}\right)$. The Coulomb barrier may be a source of electron and positron pairs at finite temperature [9]. Therefore the Schwinger pair production at finite temperature would be interesting for such astrophysical objects.

It was Dittrich who first found the QED effective action at finite temperature in the presence of a constant magnetic field [10]. Since then this has motivated the study of QED at finite temperature under various configurations of electromagnetic fields and thereby the Schwinger pair production at finite temperature, which has been an issue of debate depending on formalisms employed. The QED effective action for a constant magnetic field was obtained at finite temperature and density [11]. Loewe and Rojas used the Schwinger proper-time method to obtain the

*Electronic address: sangkim@kunsan.ac.kr

${ }^{\dagger}$ Electronic address: hyunkyu@hanyang.ac.kr 
effective action in the presence of both constant magnetic and electric fields and found an imaginary part that has contributions from vacuum as well as thermal fluctuations, leading to a thermal enhancement of pair production at high temperature [12]. On the other hand, Elmfors and Skagerstam used the real-time formalism to obtain the QED effective action in the presence of both magnetic and electric fields, in which there is no imaginary part and thereby no pair production [13]. Contrary to this, Ganguly et al found in the finite temperature field theory that the pair-production rate increases at high temperature [14]. Also in the functional Schrödinger picture Hallin and Liljenberg found the density matrix for QED when the electric field is turned on only for a finite period and thereby calculated the pair production at finite temperature when the system evolves from an initial ensemble [15]. Recently Gies used the imaginary-time formalism to obtain the QED effective action in the presence of both constant electric and magnetic fields, which does not have any imaginary part at one-loop but nonzero imaginary part at two-loop [16].

The main purpose of this paper is to study the Schwinger pair production from an initial ensemble of charged bosons in scalar QED when an electric field is turned on only for a finite period together with or without a constant magnetic field. A Sauter-type electric field $E(t)=E_{0} \operatorname{sech}^{2}(t / \tau)$ provides an analytical model [17], which acts effectively for $-\tau \leq t \leq \tau$. The pair production from vacuum by this profile of electric field extended in a finite time or space has been studied [18, 19, 20, 21, 22, 23, 24]. Note that as the gauge potential $A_{\mu}=\left(0,0,0,-E_{0} \tau \tanh (t / \tau)\right)$ in the time-dependent gauge depends on time explicitly, so does the Hamiltonian. As the evolution operator $e^{-\beta H}$ in the Euclidean time is not the density operator, the imaginary-time method based on the Matsubara frequency may not be used directly for general time-dependent cases. In fact, the initial ensemble evolves out of equilibrium due to the time-dependent Hamiltonian, which requires a nonequilibrium quantum field theory.

In this paper we shall use a nonequilibrium quantum field theory based on the Liouville-von Neumann equation, which was originally introduced to find the density operator but led to exact quantum states of time-dependent systems [25, 26]. The scalar QED Hamiltonian is quadratic in the field and momentum and each momentum mode has a time-dependent frequency. The time-dependent creation and annihilation operators that satisfy the Liouvillevon Neumann equation provide not only the density operator but also exact quantum states. We find that the number of pairs produced by the electric field is proportional to the initial Bose-Einstein distribution. From this scalar QED model we may conclude that the Schwinger pair-production rate by a time-dependent electric field is enhanced by a thermal factor of the initial Bose-Einstein distribution. The Liouville-von Neumann method can be compared with other methods used to handle the scalar QED Hamiltonian. Hallin and Liljenberg used the Schrödinger picture to find the wave functional of the Schrödinger equation and the density matrix [15], whereas the authors of Refs. 227, 28] used the Heisenberg picture to find the time evolution of the creation and annihilation operators for each momentum of the field. It should be remarked that the time-dependent creation and annihilation operators of the Liouville-von Neumann equation provide not only the density operator but also exact quantum states for each momentum.

The organization of this paper is as follows. In Sec. II, we explain the canonical quantization method based on the Liouville-von Neumann equation and apply it to scalar QED in the presence of an electric field. In Sec. III, we calculate the Schwinger pair-production rate at finite temperature in the presence of Sauter-type electric field together with or without a constant magnetic field.

\section{CANONICAL QUANTIZATION OF SCALAR QED}

Scalar QED for a charged boson with mass $m$ and charge $q$ under an external electromagnetic field with the gauge field $A_{\mu}$ is described by the Lagrangian [in units with $\hbar=c=1$ and with metric signature $(+,-,-,-)$ ]

$$
L=\int d^{3} x\left[\eta^{\mu \nu}\left(\nabla_{\mu} \Phi\right)^{*}\left(\nabla_{\nu} \Phi\right)-m^{2} \Phi^{*} \Phi\right]
$$

where the covariant derivative is given by

$$
\nabla_{\mu}=\partial_{\mu}+i q A_{\mu}
$$

For instance, the electric field in the $z$-direction may be described by the time-dependent gauge potential $A_{\mu}=$ $\left(0,0,0, A_{z}\right)$. Then the Klein-Gordon equation from the Lagrangian (1) takes the form

$$
\left[\partial_{t}^{2}-\partial_{\perp}^{2}-\left(\partial_{z}+i q A_{z}(t)\right)^{2}+m^{2}\right] \Phi=0 .
$$

The corresponding Hamiltonian is given by

$$
H=\int d^{3} x\left[\Pi^{*} \Pi+\Phi^{*}\left(-\partial_{\perp}^{2}-\left(\partial_{z}+i q A_{z}\right)^{2}+m^{2}\right) \Phi\right]
$$


where $\Pi=\dot{\Phi}^{*}$ and $\Pi^{*}=\dot{\Phi}$ are the conjugate momenta.

Following Ref. [26], the fields and momenta are decomposed into Fourier modes as

$$
\begin{aligned}
\Phi(\mathbf{x}, t) & =\int[d \mathbf{k}] \phi_{\mathbf{k}} e^{i \mathbf{k} \cdot \mathbf{x}}, \\
\Phi^{*}(\mathbf{x}, t) & =\int[d \mathbf{k}] \phi_{\mathbf{k}}^{*} e^{-i \mathbf{k} \cdot \mathbf{x}},
\end{aligned}
$$

where $[d \mathbf{k}]=d^{3} \mathbf{k} /(2 \pi)^{3}$ and

$$
\begin{aligned}
\Pi(\mathbf{x}, t) & =\int[d \mathbf{k}] \dot{\phi}_{\mathbf{k}}^{*} e^{-i \mathbf{k} \cdot \mathbf{x}}=\int[d \mathbf{k}] \pi_{\mathbf{k}} e^{-i \mathbf{k} \cdot \mathbf{x}}, \\
\Pi^{*}(\mathbf{x}, t) & =\int[d \mathbf{k}] \dot{\phi}_{\mathbf{k}} e^{i \mathbf{k} \cdot \mathbf{x}}=\int[d \mathbf{k}] \pi_{\mathbf{k}}^{*} e^{i \mathbf{k} \cdot \mathbf{x}}
\end{aligned}
$$

The Hamiltonian is then given by

$$
H=\int[d \mathbf{k}]\left[\pi_{\mathbf{k}}^{*} \pi_{\mathbf{k}}+\omega_{\mathbf{k}}^{2}(t) \phi_{\mathbf{k}}^{*} \phi_{\mathbf{k}}\right],
$$

where

$$
\omega_{\mathbf{k}}^{2}(t)=\left(k_{z}+q A_{z}(t)\right)^{2}+\mathbf{k}_{\perp}^{2}+m^{2}
$$

Here, $\phi_{\mathbf{k}}, \phi_{\mathbf{k}}^{*}$ and $\pi_{\mathbf{k}}, \pi_{\mathbf{k}}^{*}$ are Schrödinger operators. Each Fourier mode obeys the equation

$$
\left[\partial_{t}^{2}+\omega_{\mathbf{k}}^{2}(t)\right] \phi_{\mathbf{k}}(t)=0
$$

Upon canonical quantization, we impose the equal-time commutation relation $\left[\Phi\left(\mathbf{x}^{\prime}, t\right), \Pi(\mathbf{x}, t)\right]=i \delta\left(\mathbf{x}^{\prime}-\mathbf{x}\right)$ and $\left[\Phi^{*}\left(\mathbf{x}^{\prime}, t\right), \Pi^{*}(\mathbf{x}, t)\right]=i \delta\left(\mathbf{x}^{\prime}-\mathbf{x}\right)$, from which follow the commutation relations

$$
\begin{aligned}
{\left[\phi_{\mathbf{k}^{\prime}}, \pi_{\mathbf{k}}\right] } & =i(2 \pi)^{3} \delta\left(\mathbf{k}^{\prime}-\mathbf{k}\right), \\
{\left[\phi_{\mathbf{k}^{\prime}}^{*}, \pi_{\mathbf{k}}^{*}\right] } & =i(2 \pi)^{3} \delta\left(\mathbf{k}^{\prime}-\mathbf{k}\right),
\end{aligned}
$$

but all the other commutators vanish. We may introduce the time-dependent annihilation and creation operators to quantize the fields as

$$
\begin{aligned}
\Phi(\mathbf{x}, t) & =\int[d \mathbf{k}]\left[\varphi_{\mathbf{k}}(t) a_{\mathbf{k}}(t)+\varphi_{\mathbf{k}}^{*}(t) b_{\mathbf{k}}^{\dagger}(t)\right] e^{i \mathbf{k} \cdot \mathbf{x}}, \\
\Phi^{*}(\mathbf{x}, t) & =\int[d \mathbf{k}]\left[\varphi_{\mathbf{k}}(t) b_{\mathbf{k}}(t)+\varphi_{\mathbf{k}}^{*}(t) a_{\mathbf{k}}^{\dagger}(t)\right] e^{-i \mathbf{k} \cdot \mathbf{x}},
\end{aligned}
$$

and similarly the momenta as

$$
\begin{aligned}
\Pi(\mathbf{x}, t) & =\int[d \mathbf{k}]\left[\dot{\varphi}_{\mathbf{k}}^{*}(t) a_{\mathbf{k}}^{\dagger}(t)+\dot{\varphi}_{\mathbf{k}}(t) b_{\mathbf{k}}(t)\right] e^{-i \mathbf{k} \cdot \mathbf{x}}, \\
\Pi^{*}(\mathbf{x}, t) & =\int[d \mathbf{k}]\left[\dot{\varphi}_{\mathbf{k}}^{*}(t) b_{\mathbf{k}}^{\dagger}(t)+\dot{\varphi}_{\mathbf{k}}(t) a_{\mathbf{k}}(t)\right] e^{i \mathbf{k} \cdot \mathbf{x}} .
\end{aligned}
$$

A few comments are in order. First, note that $a_{\mathbf{k}}$ and $a_{\mathbf{k}}^{\dagger}$ correspond to the particle and $b_{\mathbf{k}}$ and $b_{\mathbf{k}}^{\dagger}$ to the antiparticle and that $\Phi$ annihilates particles but creates antiparticles. Second, it can be shown that the field quantization includes static fields as a specific case by choosing $\varphi_{\mathbf{k}}=e^{-i \omega_{\mathbf{k}} t} / \sqrt{2 \omega_{\mathbf{k}}}$. Finally, by imposing the Wronskian conditions

$$
\dot{\varphi}_{\mathbf{k}}^{*}(t) \varphi_{\mathbf{k}}(t)-\varphi_{\mathbf{k}}^{*}(t) \dot{\varphi}_{\mathbf{k}}(t)=i
$$

we have the standard equal-time commutation relations

$$
\left[a_{\mathbf{k}^{\prime}}(t), a_{\mathbf{k}}^{\dagger}(t)\right]=\delta\left(\mathbf{k}^{\prime}-\mathbf{k}\right), \quad\left[b_{\mathbf{k}^{\prime}}(t), b_{\mathbf{k}}^{\dagger}(t)\right]=\delta\left(\mathbf{k}^{\prime}-\mathbf{k}\right)
$$

All the other commutators vanish. 
By inverting the Fourier modes in Eqs. (11) and (12), we find two independent sets of time-dependent annihilation and creation operators [26]

$$
\begin{aligned}
& a_{\mathbf{k}}(t)=i\left[\varphi_{\mathbf{k}}^{*}(t) \pi_{\mathbf{k}}^{*}-\dot{\varphi}_{\mathbf{k}}^{*}(t) \phi_{\mathbf{k}}\right] \\
& a_{\mathbf{k}}^{\dagger}(t)=-i\left[\varphi_{\mathbf{k}}(t) \pi_{\mathbf{k}}-\dot{\varphi}_{\mathbf{k}}(t) \phi_{\mathbf{k}}^{*}\right]
\end{aligned}
$$

and

$$
\begin{aligned}
& b_{\mathbf{k}}(t)=i\left[\varphi_{\mathbf{k}}^{*}(t) \pi_{\mathbf{k}}-\dot{\varphi}_{\mathbf{k}}^{*}(t) \phi_{\mathbf{k}}^{*}\right] \\
& b_{\mathbf{k}}^{\dagger}(t)=-i\left[\varphi_{\mathbf{k}}(t) \pi_{\mathbf{k}}^{*}-\dot{\varphi}_{\mathbf{k}}(t) \phi_{\mathbf{k}}\right] .
\end{aligned}
$$

It is remarkable that when $\varphi_{\mathbf{k}}$ satisfies the mode equation (9), the time-dependent operators (15) and (16) satisfy the Liouville-von Neumann equation, for instance,

$$
i \frac{\partial a_{\mathbf{k}}(t)}{\partial t}+\left[a_{\mathbf{k}}(t), H(t)\right]=0 .
$$

This implies that we may define the density operator for particles

$$
\rho_{a_{\mathbf{k}}}(t)=\frac{1}{Z_{\mathbf{k}}} \exp \left[-\frac{\omega_{\mathbf{k}}}{k T}\left(a_{\mathbf{k}}^{\dagger}(t) a_{\mathbf{k}}(t)+\frac{1}{2}\right)\right]
$$

and for antiparticles

$$
\rho_{b_{\mathbf{k}}}(t)=\frac{1}{Z_{\mathbf{k}}} \exp \left[-\frac{\omega_{\mathbf{k}}}{k T}\left(b_{\mathbf{k}}^{\dagger}(t) b_{\mathbf{k}}(t)+\frac{1}{2}\right)\right] .
$$

Here, $Z_{\mathbf{k}}=\operatorname{Tr}\left(e^{-\omega_{\mathbf{k}}\left(a_{\mathbf{k}}^{\dagger}(t) a_{\mathbf{k}}(t)+1 / 2\right) / k T}\right)=\operatorname{Tr}\left(e^{-\omega_{\mathbf{k}}\left(b_{\mathbf{k}}^{\dagger}(t) b_{\mathbf{k}}(t)+1 / 2\right) / k T}\right)$. We note that $T$ is the temperature of an initial ensemble of the heat bath of particles before the onset of electric fields and that the number of produced pairs should be small not to change the temperature of heat bath.

We now turn to pair production by electric fields. The mode-decomposed Hamiltonian (7) is a collection of timedependent harmonic oscillators. The equation of motion (9), in the language of quantum mechanics, becomes the super-barrier transmission over a potential barrier in the time dimension. Pair production may be explained in such a way that the positive-frequency solution at the past infinity scatters not only to the positive-frequency solution but also to the negative-frequency solution at the future infinity. Then the magnitude square of the ratio of the coefficient of the negative-frequency solution at the future infinity to the coefficient of the positive-frequency solution at the past infinity is the pair-production rate [29, 30]. However, we shall employ the Liouville-von Neumann method, in which we first find the operators known as the Lewis-Riesenfeld or invariant operators that satisfy the Liouville-von Neumann equation [25] and then construct not only the exact quantum states but also the density operator. The time-dependent vacuum is defined as

$$
a_{\mathbf{k}}(t)|0 ; t\rangle=b_{\mathbf{k}}(t)|0 ; t\rangle=0, \quad(\forall \mathbf{k})
$$

and the multi-particle and antiparticle states as

$$
\left|n_{\mathbf{k}_{1}} \cdots ; n_{\mathbf{k}_{2}} \cdots ; t\right\rangle=\frac{a_{\mathbf{k}_{1}}^{\dagger n_{1}}(t)}{\sqrt{n_{1} !}} \cdots \frac{b_{\mathbf{k}_{2}}^{\dagger n_{2}}(t)}{\sqrt{n_{2} !}} \cdots|0 ; t\rangle .
$$

The time-dependent vacuum (20) is the zero-particle and antiparticle state of the number operators

$$
N_{a_{\mathbf{k}}}(t)=a_{\mathbf{k}}^{\dagger}(t) a_{\mathbf{k}}(t), \quad N_{b_{\mathbf{k}}}(t)=b_{\mathbf{k}}^{\dagger}(t) b_{\mathbf{k}}(t) .
$$

It should be remarked that the Fock states are exact quantum states of the time-dependent Hamiltonian up to some time-dependent phase factors a la the Liouville-von Neumann method [25, 26].

To compare with the standard quantization of field, we consider the limiting case of no electromagnetic field. Then our definition of the time-dependent annihilation operator differs from the standard one by an overall time-dependent phase factor:

$$
a_{\mathbf{k}}(t)=e^{i \omega_{\mathbf{k}} t} a_{\mathbf{k}}
$$


for the choice of $\varphi_{\mathbf{k}}=e^{-i \omega_{\mathbf{k}} t} / \sqrt{2 \omega_{\mathbf{k}}}$. The phase factor, which is necessary for the Liouville-von Neumann equation, does not change the number operators $N_{a_{\mathbf{k}}}(t)=N_{a_{\mathbf{k}}}$ and $N_{b_{\mathbf{k}}}(t)=N_{b_{\mathbf{k}}}$. In this sense the Liouville-von Neumann method includes the standard quantization as a specific case. The method turns out to be convenient and powerful for time-dependent quantum fields such as scalar QED in the presence of time-dependent electromagnetic fields. The number of pairs with a certain momentum $\mathbf{k}$ produced by an external field is the number of particles or antiparticles of the out-vacuum at $t=\infty$ contained in the in-vacuum at $t=-\infty[29,30$ ]

$$
n_{\mathbf{k}}=\left\langle 0 ; \operatorname{in}\left|N_{a_{\mathbf{k}}}(t=\infty)\right| 0 ; \text { in }\right\rangle=\left\langle 0 ; \text { in }\left|N_{b_{\mathbf{k}}}(t=\infty)\right| 0 ; t=\text { in }\right\rangle .
$$

\section{PAIR PRODUCTION AT FINITE TEMPERATURE}

We first study the Schwinger pair production by a uniform field $E(t)=E_{0} \operatorname{sech}^{2}(t / \tau)$ without a magnetic field at finite temperature and then study the case with a constant magnetic field $B$. When $\mathbf{E} \cdot \mathbf{B} \neq 0$ we can find a Lorentz frame where the electric and magnetic fields are parallel to each other, for instance, in the $z$-direction. We choose is a Sauter-type gauge potential $A_{\mu}=\left(0,-B y / 2, B x / 2,-E_{0} \tau \tanh (t / \tau)\right)$. It is shown that pair production by the Sauter-type potential is characterized by two parameters [22]

$$
\epsilon_{t}=\frac{m}{q E_{0} \tau}, \quad \delta_{t}=\frac{q E_{0}}{\pi m^{2}} .
$$

The pair production is energetically favored for $\epsilon_{t}<1$ and the adiabaticity parameter $\delta_{t}$ determines the pair-production rate.

\section{A. Pure Electric Field}

The mode equation for the auxiliary field variable takes the form

$$
\ddot{\varphi}_{\mathbf{k}}(t)+\omega_{\mathbf{k}}^{2}(t) \varphi_{\mathbf{k}}(t)=0
$$

where

$$
\omega_{\mathbf{k}}^{2}(t)=\left(k_{z}-q E_{0} \tau \tanh \left(\frac{t}{\tau}\right)\right)^{2}+\mathbf{k}_{\perp}^{2}+m^{2} .
$$

Each mode has two asymptotic frequencies at $t=\mp \infty$

$$
\begin{aligned}
& \omega_{\mathbf{k}}^{(-)}=\sqrt{\left(k_{z}+q E_{0} \tau\right)^{2}+\mathbf{k}_{\perp}^{2}+m^{2}}, \\
& \omega_{\mathbf{k}}^{(+)}=\sqrt{\left(k_{z}-q E_{0} \tau\right)^{2}+\mathbf{k}_{\perp}^{2}+m^{2}} .
\end{aligned}
$$

The solution with the asymptotic form

$$
\varphi_{\mathbf{k}}^{\mathrm{in}}(t)=\frac{e^{-i \omega_{\mathbf{k}}^{(-)} t}}{\sqrt{2 \omega_{\mathbf{k}}^{(-)}}}
$$

at $t=-\infty$ is given by [18, 19]

$$
\varphi_{\mathbf{k}}(t)=\frac{1}{\sqrt{2 \omega_{\mathbf{k}}^{(-)} e^{\pi \tau \omega_{\mathbf{k}}^{(-)}}}}(1-z)^{1 / 2+i \lambda} z^{-i \tau \omega_{\mathbf{k}}^{(-)} / 2} F\left(\alpha_{\mathbf{k}}, \beta_{\mathbf{k}} ; \gamma_{\mathbf{k}} ; z\right),
$$

where $F\left(\alpha_{\mathbf{k}}, \beta_{\mathbf{k}} ; \gamma_{\mathbf{k}} ; z\right)$ is the hypergeometric function and

$$
\begin{aligned}
z & =-2 e^{2 t / \tau} \\
\lambda & =\sqrt{\left(q E_{0} \tau^{2}\right)^{2}-\frac{1}{4}}
\end{aligned}
$$


and

$$
\begin{aligned}
\alpha_{\mathbf{k}} & =\frac{1}{2}-\frac{i}{2}\left(\tau \omega_{\mathbf{k}}^{(-)}-\tau \omega_{\mathbf{k}}^{(+)}-2 \lambda\right), \\
\beta_{\mathbf{k}} & =\frac{1}{2}-\frac{i}{2}\left(\tau \omega_{\mathbf{k}}^{(-)}+\tau \omega_{\mathbf{k}}^{(+)}-2 \lambda\right), \\
\gamma_{\mathbf{k}} & =1-i \tau \omega_{\mathbf{k}}^{(-)} .
\end{aligned}
$$

Note that the solution (30) satisfies the Wronskian condition (13) for any time. At the other asymptotic limit $t=\infty$, the solution becomes

$$
\varphi_{\mathbf{k}}^{(+)}(t)=A_{\mathbf{k}} \varphi_{\mathbf{k}}^{\text {out }}(t)+B_{\mathbf{k}} \varphi_{\mathbf{k}}^{\text {out* }}(t)
$$

where

$$
\varphi_{\mathbf{k}}^{\text {out }}(t)=\frac{e^{-i \omega_{\mathbf{k}}^{(+)} t}}{\sqrt{2 \omega_{\mathbf{k}}^{(+)}}}
$$

and

$$
\begin{aligned}
& A_{\mathbf{k}}=2^{-i \tau \omega_{\mathbf{k}}^{(+)}} \sqrt{\frac{\omega_{\mathbf{k}}^{(+)}}{\omega_{\mathbf{k}}^{(-)}}} \frac{\Gamma\left(\gamma_{\mathbf{k}}\right) \Gamma\left(\beta_{\mathbf{k}}-\alpha_{\mathbf{k}}\right)}{\Gamma\left(\beta_{\mathbf{k}}\right) \Gamma\left(\gamma_{\mathbf{k}}-\alpha_{\mathbf{k}}\right)}, \\
& B_{\mathbf{k}}=2^{i \tau \omega_{\mathbf{k}}^{(+)}} \sqrt{\frac{\omega_{\mathbf{k}}^{(+)}}{\omega_{\mathbf{k}}^{(-)}}} \frac{\Gamma\left(\gamma_{\mathbf{k}}\right) \Gamma\left(\alpha_{\mathbf{k}}-\beta_{\mathbf{k}}\right)}{\Gamma\left(\alpha_{\mathbf{k}}\right) \Gamma\left(\gamma_{\mathbf{k}}-\beta_{\mathbf{k}}\right)}
\end{aligned}
$$

As the asymptotic form (33) is approximately valid for $t \geq \tau$, the analysis below is a good approximation after $\tau$ and exact at $t=\infty$.

The operators in Eqs. (15) and (16) obtained by substituting the asymptotic solution (29) define the time-dependent annihilation and creation operators $a_{\mathbf{k}}^{\text {in }}, a_{\mathbf{k}}^{\dagger \text { in }}$ and $b_{\mathbf{k}}^{\text {in }}, b_{\mathbf{k}}^{\dagger \text { in }}$ for the in-vacuum while those obtained by the asymptotic solution (34) define the time-dependent annihilation and creation operators $a_{\mathbf{k}}^{\text {out }}, a_{\mathbf{k}}^{\text {tout }}$ and $b_{\mathbf{k}}^{\text {out }}, b_{\mathbf{k}}^{\text {tout }}$ for the outvacuum. The mode solution starting from the initial asymptotic solution (29) evolves to another asymptotic form (33). This leads to a Bogoliubov transformation between the in-vacuum operators and the out-vacuum operators

$$
\begin{aligned}
& a_{\mathbf{k}}^{\text {out }}=A_{\mathbf{k}} a_{\mathbf{k}}^{\text {in }}+B_{\mathbf{k}}^{*} b_{\mathbf{k}}^{\text {in }}, \\
& b_{\mathbf{k}}^{\text {out }}=A_{\mathbf{k}} b_{\mathbf{k}}^{\text {in }}+B_{\mathbf{k}}^{*} a_{\mathbf{k}}^{\text {†i }},
\end{aligned}
$$

whose coefficients satisfy the relation

$$
\left|A_{\mathbf{k}}\right|^{2}-\left|B_{\mathbf{k}}\right|^{2}=1
$$

The in-vacuum is the superposition of the out-vacuum particles. The number operator of the in-vacuum is $N_{a_{\mathbf{k}}}^{\text {in }}=$ $a_{\mathbf{k}}^{\dagger \text { in }} a_{\mathbf{k}}^{\text {in }}$ for particles and $N_{b_{\mathbf{k}}}^{\text {in }}=b_{\mathbf{k}}^{\dagger \text { in }} b_{\mathbf{k}}^{\text {in }}$ for the antiparticles. Similarly, the particle number operator of the out-vacuum is $N_{a_{\mathbf{k}}}^{\text {out }}=a_{\mathbf{k}}^{\text {†out }} a_{\mathbf{k}}^{\text {out }}$ and the antiparticle number operator is $N_{b_{\mathbf{k}}}^{\text {out }}=b_{\mathbf{k}}^{\text {†out }} b_{\mathbf{k}}^{\text {out }}$. Hence the number of created pairs by the electric field from the vacuum is

$$
n_{\mathbf{k}}=\left\langle 0 ; \text { in }\left|N_{a_{\mathbf{k}}}^{\text {out }}\right| 0 ; \text { in }\right\rangle=\left\langle 0 ; \text { in }\left|N_{b_{\mathbf{k}}}^{\text {out }}\right| 0 ; \text { in }\right\rangle=\left|B_{\mathbf{k}}\right|^{2},
$$

where

$$
\left|B_{\mathbf{k}}\right|^{2}=\frac{\cosh \left[\pi\left(2 \lambda+\tau \omega_{\mathbf{k}}^{(+)}-\tau \omega_{\mathbf{k}}^{(-)}\right) / 2\right] \cosh \left[\pi\left(2 \lambda+\tau \omega_{\mathbf{k}}^{(-)}-\tau \omega_{\mathbf{k}}^{(+)}\right) / 2\right]}{\cosh \left[\pi\left(2 \lambda+\tau \omega_{\mathbf{k}}^{(-)}+\tau \omega_{\mathbf{k}}^{(+)}\right) / 2\right] \cosh \left[\pi\left(2 \lambda-\tau \omega_{\mathbf{k}}^{(-)}-\tau \omega_{\mathbf{k}}^{(+)}\right) / 2\right]} .
$$

The number of pairs (39) approximately is

$$
\left|B_{\mathbf{k}}\right|^{2} \approx e^{-\pi\left(\tau \omega_{\mathbf{k}}^{(-)}+\tau \omega_{\mathbf{k}}^{(+)}-\lambda\right)}=e^{-S_{\mathbf{k}}^{(0)}}
$$


where $S_{\mathbf{k}}^{(0)}$ is the leading-order contribution of the WKB instanton action in scalar QED [22].

The density operator (18) takes the asymptotic form for particles

$$
\rho_{a_{\mathbf{k}}}^{\text {in }}=\frac{1}{Z_{\mathbf{k}}} \exp \left[-\frac{\omega_{\mathbf{k}}^{(-)}}{k T}\left(N_{a_{\mathbf{k}}}^{\text {in }}+\frac{1}{2}\right)\right]
$$

and the density operator (19) for antiparticles

$$
\rho_{b_{\mathbf{k}}}^{\text {in }}=\frac{1}{Z_{\mathbf{k}}} \exp \left[-\frac{\omega_{\mathbf{k}}^{(-)}}{k T}\left(N_{b_{\mathbf{k}}}^{\text {in }}+\frac{1}{2}\right)\right] .
$$

Hence the initial ensemble of particles has the Bose-Einstein distribution

$$
f_{\mathbf{k}}(T)=\operatorname{Tr}\left(\rho_{\mathbf{k}}^{\text {in }} N_{a_{\mathbf{k}}}^{\text {in }}\right)=\frac{1}{e^{\omega_{\mathbf{k}}^{(-)} / k T}-1} .
$$

Finally, the number of pairs produced by the electric field from the initial thermal ensemble is

$$
n_{\mathbf{k}}(E, T)=\operatorname{Tr}\left(\rho_{\mathbf{k}}^{\text {in }} N_{a_{\mathbf{k}}}^{\text {out }}\right)-f_{\mathbf{k}}=\left|B_{\mathbf{k}}(E)\right|^{2}\left(2 f_{\mathbf{k}}(T)+1\right) .
$$

The thermal factor can be written as $2 f_{\mathbf{k}}+1=\operatorname{coth}\left(\omega_{\mathbf{k}}^{(-)} / 2 k T\right)$. We have thus found that the Schwinger pairproduction rate at finite temperature is enhanced by the thermal factor $f_{\mathbf{k}}$. In the zero-temperature limit we have $f_{\mathbf{k}}=0$ and recover the Schwinger pair-production rate from the vacuum. The Schwinger pair-production rate per unit volume and per unit time is given by summing over all momenta

$$
\mathcal{N}(E, T)=\int[d \mathbf{k}] n_{\mathbf{k}}(E, T) .
$$

As the solution (30) approximately approaches the asymptotic form (29) for $t \approx-\tau$ and (33) for $t \approx \tau$, the pairproduction rates (44) and (45) are approximately valid after $t=\tau$.

In the asymptotic region where the electric field is turned off, the out-vacuum is well-defined and the excited states (21) correspond to a number of particles and/or antiparticles. In this region the pair-production rate (44) or (45) has the interpretation of created pairs. Now a question may be raised how to interpret Eqs. (44) or (45) while the electric field is acting. In terms of the in-vacuum operators we may express the time-dependent annihilation operator at any time as

$$
\begin{aligned}
a_{\mathbf{k}}(t) & =A_{\mathbf{k}}(t) a_{\mathbf{k}}^{\text {in }}+B_{\mathbf{k}}^{*}(t) b_{\mathbf{k}}^{\text {in } \dagger}, \\
b_{\mathbf{k}}(t) & =A_{\mathbf{k}}(t) b_{\mathbf{k}}^{\text {in }}+B_{\mathbf{k}}^{*}(t) a_{\mathbf{k}}^{\text {in } \dagger},
\end{aligned}
$$

where

$$
\begin{aligned}
& A_{\mathbf{k}}(t)=i\left(\varphi_{\mathbf{k}}(t) \dot{\varphi}_{\mathbf{k}}^{\text {in }}-\dot{\varphi}_{\mathbf{k}}^{*}(t) \varphi_{\mathbf{k}}^{\text {in }}\right), \\
& B_{\mathbf{k}}(t)=i\left(\varphi_{\mathbf{k}}^{*}(t) \dot{\varphi}_{\mathbf{k}}^{\text {in } *}-\dot{\varphi}_{\mathbf{k}}^{*}(t) \varphi_{\mathbf{k}}^{\text {in }}\right) .
\end{aligned}
$$

Here $\varphi_{\mathbf{k}}^{\text {in }}$ is the solution at $t=-\infty$. Then we can obtain

$$
n_{\mathbf{k}}(E, T ; t)=\operatorname{Tr}\left(\rho_{\mathbf{k}}^{\mathrm{in}} N_{a_{\mathbf{k}}}(t)\right)-f_{\mathbf{k}}=\left|B_{\mathbf{k}}(E ; t)\right|^{2}\left(2 f_{\mathbf{k}}(T)+1\right) .
$$

It is tempting to interpret (48) as the number of particles at time $t$. However, the concept of particle can have a meaning only in the adiabatic limit where

$$
\varphi_{\mathbf{k}}^{\mathrm{ad}}(t)=\frac{e^{-i \int \omega_{\mathbf{k}}(t)}}{\sqrt{2 \omega_{\mathbf{k}}(t)}}
$$

is an approximate solution to the mode equation (26). The solution is approximately good for a large momentum since $\omega_{\mathbf{k}} \gg\left|\dot{\omega}_{\mathbf{k}}\right|,\left|\ddot{\omega}_{\mathbf{k}}\right|$. For a small momentum the change of the gauge potential and the electric field should be small to guarantee the validity of the adiabatic solution. In the adiabatic case the solution with the initial asymptotic form (29) evolves approximately to the form

$$
\varphi_{\mathbf{k}}(t) \approx A_{\mathbf{k}}(t) \varphi_{\mathbf{k}}^{\mathrm{ad}}(t)+B_{\mathbf{k}}(t) \varphi_{\mathbf{k}}^{\mathrm{ad} *}(t) .
$$

In the other case where the adiabatic approximation breaks down, we cannot have a concept of particle for such momentum modes but Eq. (48) may be interpreted as a distribution. 


\section{B. Electric and Magnetic Fields}

We now consider the case of a uniform field $E(t)=E_{0} \operatorname{sech}^{2}(t / \tau)$ together with a parallel magnetic field $B$. The gauge potential $A_{\mu}=\left(0,-B y / 2, B x / 2,-E_{0} \tau \tanh (t / \tau)\right)$. The mode equation takes the form

$$
\ddot{\varphi}_{k}(t, x, y)+\omega_{k}^{2}(t) \varphi_{k}(t, x, y)=0
$$

where

$$
\omega_{k}^{2}(t)=H_{\perp}+\left(k_{z}-q E_{0} \tau \tanh \left(\frac{t}{\tau}\right)\right)^{2}+m^{2} .
$$

Here $H_{\perp}$ describes the two-dimensional motion transverse to the magnetic field

$$
\begin{aligned}
H_{\perp} & =-\left(\partial_{x}-i \frac{q B}{2} y\right)^{2}-\left(\partial_{y}+i \frac{q B}{2} x\right)^{2} \\
& =-\left(\partial_{x}^{2}+\partial_{y}^{2}\right)+\left(\frac{q B}{2}\right)^{2}\left(x^{2}+y^{2}\right)-\frac{q B}{2} L_{z} .
\end{aligned}
$$

It has the eigenvalue

$$
H_{\perp} \varphi_{n}(x, y)=q B(2 n+1) \varphi_{n}(x, y),
$$

whose orthonormal eigenfunctions were given in Ref. [31]. Then the mode $\varphi_{k}(t, x, y)=\varphi_{n k}(t) \varphi_{n}(x, y)$ satisfies

$$
\ddot{\varphi}_{n k}(t)+\omega_{n k}^{2}(t) \varphi_{n k}(t)=0,
$$

where

$$
\omega_{n k}^{2}=\left(k_{z}-q E_{0} \tau \tanh \left(\frac{t}{\tau}\right)\right)^{2}+q B(2 n+1)+m^{2} .
$$

The two asymptotic frequencies at $t=\mp \infty$ are given by

$$
\begin{aligned}
& \omega_{n k}^{(-)}=\sqrt{\left(k_{z}+q E_{0} \tau\right)^{2}+q B(2 n+1)+m^{2}}, \\
& \omega_{n k}^{(+)}=\sqrt{\left(k_{z}-q E_{0} \tau\right)^{2}+q B(2 n+1)+m^{2}} .
\end{aligned}
$$

The mode has the solution of the same form as Eq. (30)

$$
\varphi_{n k}(t)=\frac{1}{\sqrt{2 \omega_{n k}^{(-)} e^{\pi \tau \omega_{n k}^{(-)}}}}(1-z)^{1 / 2+i \lambda} z^{-i \tau \omega_{n k}^{(-)} / 2} F\left(\alpha_{n k}, \beta_{n k} ; \gamma_{n k} ; z\right),
$$

with parameters replaced by

$$
\begin{aligned}
\alpha_{n k} & =\frac{1}{2}-\frac{i}{2}\left(\tau \omega_{n k}^{(-)}-\tau \omega_{n k}^{(+)}-2 \lambda\right), \\
\beta_{n k} & =\frac{1}{2}-\frac{i}{2}\left(\tau \omega_{n k}^{(-)}+\tau \omega_{n k}^{(+)}-2 \lambda\right), \\
\gamma_{n k} & =1-i \tau \omega_{n k}^{(-)} .
\end{aligned}
$$

The Bogoliubov transformations for particles and antiparticles are given by

$$
\begin{aligned}
& a_{n k}^{\text {out }}=A_{n k} a_{n k}^{\text {in }}+B_{n k}^{*} b_{n k}^{\text {in }}, \\
& b_{n k}^{\text {out }}=A_{n k} b_{n k}^{\text {in }}+B_{n k}^{*} a_{n k}^{\text {in }},
\end{aligned}
$$

where

$$
\begin{aligned}
& A_{n k}=2^{-i \tau \omega_{n k}^{(+)}} \sqrt{\frac{\omega_{n k}^{(+)}}{\omega_{n k}^{(-)}}} \frac{\Gamma\left(\gamma_{n k}\right) \Gamma\left(\beta_{n k}-\alpha_{n k}\right)}{\Gamma\left(\beta_{n k}\right) \Gamma\left(\gamma_{n k}-\alpha_{n k}\right)}, \\
& B_{n k}=2^{i \tau \omega_{n k}^{(+)}} \sqrt{\frac{\omega_{n k}^{(+)}}{\omega_{n k}^{(-)}} \frac{\Gamma\left(\gamma_{n k}\right) \Gamma\left(\alpha_{n k}-\beta_{n k}\right)}{\Gamma\left(\alpha_{n k}\right) \Gamma\left(\gamma_{n k}-\beta_{n k}\right)}} .
\end{aligned}
$$


where

$$
f_{n k}(T)=\operatorname{Tr}\left(\rho_{n k}^{\mathrm{in}} N_{a_{n k}}^{\mathrm{in}}\right)=\frac{1}{e^{\omega_{n k}^{(-)} / k T}-1} .
$$

Finally, as in the pure electric field case, we find the number of pairs produced from the initial thermal ensemble

$$
n_{n k}(E, B, T)=\operatorname{Tr}\left(\rho_{n k}^{\text {in }} N_{a_{n k}}^{\text {out }}\right)-f_{n k}=\left|B_{n k}(E, B)\right|^{2}\left(2 f_{n k}(T)+1\right) .
$$

In the case of a pure magnetic field, $\alpha_{n k}=0$ and thereby $B_{n k}=0$ as $\Gamma\left(\alpha_{n k}=0\right)=\infty$ and $A_{n k}=e^{-i \tau \omega_{n k}}$ becomes a pure phase factor. This confirms the fact that pure magnetic fields cannot produce pairs of particles and antiparticles even at finite temperature. The total number of pairs per unit volume and per unit time is given by summing over the Landau levels and the longitudinal momentum

$$
\mathcal{N}(E, B, T)=\frac{q B}{(2 \pi)^{2}} \sum_{n} \int d k_{z}\left|B_{n k}(E, B)\right|^{2}\left(2 f_{n k}(T)+1\right)
$$

Here $(q B) /(2 \pi)$ is the number of Landau levels and another factor $1 /(2 \pi)$ is from the $k_{z}$ integration.

\section{CONCLUSION}

In this paper we have studied the pair production of charged bosons at finite temperature by an electric field together with or without a constant magnetic field. As an analytically solvable model, the electric field of Sautertype is used, which is effectively turned on for a finite period of time. Upon mode-decomposition the scalar QED Hamiltonian becomes a collection of time-dependent oscillators. We used the Liouville-von Neumann method to find the exact quantum states as well as the density operator for each momentum. The time-dependent gauge potential for the Sauter-type electric field has two asymptotic regions where the in-vacuum and out-vacuum are well-defined. The in-vacuum, which is annulled by the particle and antiparticle annihilation operators for each momentum before the onset of the electric field, contains a number of particles and antiparticles of the same momentum after the electric field is turned on and thus leads to pair production by the electric field. We have found that the number of created pairs for each momentum at the finite temperature is $n_{n k}(E, B, T)=n_{n k}(E, B, 0) \operatorname{coth}\left(\omega_{k}^{(-)} / 2 k T\right)$, the number of pairs produced from the vacuum times a thermal factor from the initial Bose-Einstein distribution. In the limit of zero temperature the number of created pairs reduces to that from the in-vacuum as expected. We also confirm that the pure magnetic field does not produce pairs even at finite temperature at one-loop level. We may thus conclude that the Schwinger pair-production rate is indeed enhanced by the thermal effect given by the Bose-Einstein distribution.

An interesting problem not handled in this paper is pair production of fermions at finite temperature by timedependent electric fields. The fermion pair production by an arbitrary time-dependent electric field was studied in Refs. [32, 33]. Also the fermion pair-production rate by the Sauter-type electric field was found in the WKB instanton action method [22] and in the worldline instanton method [23, 24]. However, to extend fermion pair production to the finite temperature case, we need a density operator for the time-dependent fermion system. For instance, the time-dependent annihilation and creation operators in Ref. 34] that satisfy the Liouville-von Neumann equation may be used to calculate the fermion pair-production rate at finite temperature. Another interesting problem is the back reaction of the produced pairs to the initial electromagnetic field. The back reaction problem in scalar and spinor QED was studied in Ref. [35]. As produced pairs can generate an additional electromagnetic field to the external field, the produced pairs provide a source term to the Klein-Gordon equation or the Dirac equation. The back reaction problem at finite temperature in scalar and spinor QED will be addressed in a future publication.

\section{Acknowledgments}

The authors would like to thank Don N. Page and Misao Sasaki for useful discussions and comments. The work of S. P. K. was supported by the Korea Science and Engineering Foundation (KOSEF) grant funded by the Korea government (MOST) (No. F01-2007-000-10188-0). The work of H. K. L was supported by the Korea Science and Engineering Foundation (KOSEF) grant funded by the Korea government (MOST) (No. R01-2006-000-10651-0).

[1] F. Sauter, Z. Phys. 69, 742 (1931); W. Heisenberg and H. Euler, Z. Physik 98, 714 (1936); V. Weisskopf, K. Dan. Vidensk. Selsk. Mat. Fys. Medd. XIV, 6 (1936). 
[2] J. Schwinger, Phys. Rev. 82, 664 (1951).

[3] G. V. Dunne, "Heisenberg-Euler Effective Lagrangians: Basics and Extensions," From Fields to Strings: Circumnavigating Theoretical Physics, edited by M. Shifman, A. Vainshtein, and J. Wheater, (World Scientific, Singapore, 2005), Vol. I, pp. 445-522, hep-th/0406216.

[4] A. Ringwald, Phys. Lett. B 510, 107 (2001); "Fundamental Physics at an X-Ray Free Electron Laser," Electromagnetic Probes of Fundamental Physics, edited by W. Marciano and S. White (World Scientific, Singapore, 2003), pp. 63-74, hep-ph/0112254 "Boiling the Vacuum with an X-Ray Free Electron Laser," Quantum Aspects of Beam Physics 2003, edited by P. Chen and K. Reil (World Scientific, Singapore, 2004), pp. 149-163, hep-ph/0304139

[5] SLAC Linac Coherent Light Source, 2007, http://www-ssrl.slac.stanford.edu/lcls

[6] DESY X-Ray Free Electron Laser, 2007, http://xfel.desy.de.

[7] E. Witten, Phys. Rev. D 30, 272 (1984); E. Farhi and R. L. Jaffe, Phys. Rev. D 30, 2379 (1984).

[8] P. Haensel, J. L. Zdunik, and R. Schaeffer, Astron. Astrophys. 160, 121 (1986); C. Alcock, E. Farhi, and A. Olinto, Astrophys. J. 310, 261 (1986).

[9] V. V. Usov, Phys. Rev. Lett. 80, 230 (1998); Astrophys. J. 550, L179 (2001); V. V. Usov, T. Harko, and K. S. Cheng, Astrophys. J. 620, 915 (2005); T. Harko and K. S. Cheng, Astrophys. J. 643, 318 (2006).

[10] W. Dittrich, Phys. Rev. D 19, 2385 (1979).

[11] P. Elmfors, D. Persson, and B.-S. Skagerstam, Phys. Rev. Lett. 71, 480 (1993); P. Elmfors, P. Liljenberg, D. Persson, and B.-S. Skagerstam, Phys. Rev. D 51, 5885 (1995).

[12] M. Loewe and J. C. Rojas, Phys. Rev. D 46, 2689 (1992).

[13] P. Elmfors and B.-S. Skagerstam, Phys. Lett. B 348, 141 (1995).

[14] A. K. Ganguly, P. K. Kaw, and J. C. Parikh, Phys. Rev. C 51, 2091 (1995).

[15] J. Hallin and P. Liljenberg, Phys. Rev. D 52, 1150 (1995).

[16] H. Gies, Phys. Rev. D 60, 105002 (1999); Phys. Rev. D 61, 085021 (2000).

[17] F. Sauter, Z. Phys. 73, 547 (1932).

[18] N. B. Narozhnyi and A. I. Nikishov, Yad. Fiz. 11, 1072 (1970) [Sov. J. Nucl. Phys. 11, 596 (1970)].

[19] J. Ambjorn, R. J. Hughes, and N. K. Nielsen, Ann. Phys. 150, 92 (1983).

[20] A. B. Balantekin and S. H. Fricke, Phys. Rev. D 43, 250 (1991).

[21] S. P. Kim and D. N. Page, Phys. Rev. D 65, 105002 (2002); S. P. Kim and D. N. Page, Phys. Rev. D 73, 065020 (2006).

[22] S. P. Kim and D. N. Page, Phys. Rev. D 75, 045013 (2007).

[23] G. V. Dunne and C. Schubert, Phys. Rev. D 72, 105004 (2005).

[24] G. V. Dunne, Q.-H. Wang, H. Gies, and C. Schubert, Phys. Rev. D 73, 065028 (2006).

[25] H. R. Lewis, Jr. and W. B. Riesenfeld, J. Math. Phys. 10, 1458 (1969).

[26] S. P. Kim and C. H. Lee, Phys. Rev. D 62, 125020 (2000); S. Sengupta, F. C. Khanna, and S. P. Kim, Phys. Rev. D 68, 105014 (2003).

[27] S. .M Schmidt, D. Blaschke, G. Röpke, S. A. Smolyansky, A. V. Prozorkevich, and V. D. Toneev, Int. J. Mod. Phys. E 7, 709 (1998); V. N. Pervushin, V. V. Skokov, A. V. Reichel, S. A. Smolyansky, and A. V. Prozorkevich, Int. J. Mod. Phys. A 20, 5689 (1998).

[28] S. P. Gavrilov, D. M. Gitman, and J. L. Tomazelli, hep-th/0612064

[29] L. Parker, Phys. Rev. Lett. 21, 562 (1968); Phys. Rev. 183, 1057 (1969).

[30] B. S. DeWitt, Phys. Rep. 19, 295 (1975).

[31] M. H. Johnson and B. A. Lippmann, Phys. Rev 76, 828 (1949).

[32] D. D. Dietrich, Phys. Rev. D 68, 105005 (2003).

[33] F. Cooper and G. C. Nayak, hep-th/0612292

[34] S. P. Kim, A. E. Santana, and F. C. Khanna, Phys. Lett. A 272, 46 (2000).

[35] F. Cooper and E. Mottola, Phys. Rev. D 40, 456 (1989); Y. Kluger, J. M. Eisenberg, B. Svetitsky, F. Cooper, and E. Mottola, Phys. Rev. D 45, 4659 (1992). 